\title{
Differential Lysis Approach Enables Selective Extraction of Taxon-Specific Proteins for Gut Metaproteomics
}

Jiaqin Wang ${ }^{1,2}$, Xu Zhang ${ }^{1}$, Leyuan $\mathrm{Li}^{1}$, Zhibin Ning ${ }^{1}$, Janice Mayne ${ }^{1}$, Cian SchmittUlms $^{1}$, Krystal Walker ${ }^{1}$, Kai Cheng ${ }^{1}$, Daniel Figeys ${ }^{1,3^{*}}$

1 SIMM-University of Ottawa Joint Research Center in Systems and Personalized Pharmacology and Ottawa Institute of Systems Biology and Department of Biochemistry, Microbiology and Immunology, Faculty of Medicine, University of Ottawa, Ottawa K1H 8M5, Canada.

2 College of Chemistry and Materials Science, Nanjing Normal University, Nanjing 210023, China.

3 Canadian Institute for Advanced Research, Toronto M5G 1M1, Canada.

*Corresponding author

Requests for materials should be addressed to D.F. (email: dfigeys@uottawa.ca) 
Table S1. The number of peptides identified in each step using two differential lysis (DL) methods

Table S2. Experimental conditions used for the seven-step protocol for protein extraction with method DL 2

Figure S1. The overlap of protein groups identified among the five lysis conditions using DL 1 and DL 2

Figure S2. The overlap of peptides identified among the five lysis conditions using DL 1 and DL 2

Figure S3. The change of relative abundance of several taxa in DL 1.

Figure S4. The change of relative abundance of several taxa in DL 2.

Figure S5. Evaluation of three DL methods on the taxonomic compositions in sample V46.

Figure S6. Evaluation of three DL methods on the taxonomic compositions in sample V47.

Figure S7. Evaluation of the seven-step protocol with DL 2 on the taxonomic compositions in sample V45 and V46.

Figure S8. Evaluation of three DL methods on the distributions of COG categories based on the enrichment analysis in sample V46.

Figure S9. Evaluation of three DL methods on the distributions of COG categories based on the enrichment analysis in sample V47. 
Table S1. The number of peptides identified in each step of two differential lysis (DL) methods (DL 1 and DL 2).

\begin{tabular}{|c|c|c|c|c|c|}
\hline & 1 & 2 & 3 & 4 & 5 \\
\hline DL 1 & 8234 & 5804 & 13552 & 15105 & 14869 \\
\hline DL 2 & 6379 & 4201 & 8500 & 9752 & 16572 \\
\hline
\end{tabular}


Table S2. Experimental conditions used for the seven-step protocol for protein extraction with method DL 2

\begin{tabular}{|c|c|c|c|c|}
\hline Lysis buffer & $\begin{array}{c}\text { Urea } \\
(\mathrm{M})\end{array}$ & $\begin{array}{c}\text { SDS } \\
(\% \mathrm{w} / \mathrm{v})\end{array}$ & $\begin{array}{c}\text { Tris-HCl } \\
(\mathrm{pH} 8.0)(\mathrm{mM})\end{array}$ & $\begin{array}{c}\text { Ultrasonication } \\
(\mathrm{min})\end{array}$ \\
\hline 1 & 2 & 0 & 50 & 0 \\
\hline 2 & 4 & 0 & 50 & 0 \\
\hline 3 & 8 & 0.25 & 50 & 0 \\
\hline 4 & 8 & 0.5 & 50 & 0 \\
\hline 5 & 8 & 1 & 50 & 0 \\
\hline 6 & 8 & 2 & 50 & 10 \\
\hline 7 & 8 & 4 & 50 & 0 \\
\hline
\end{tabular}




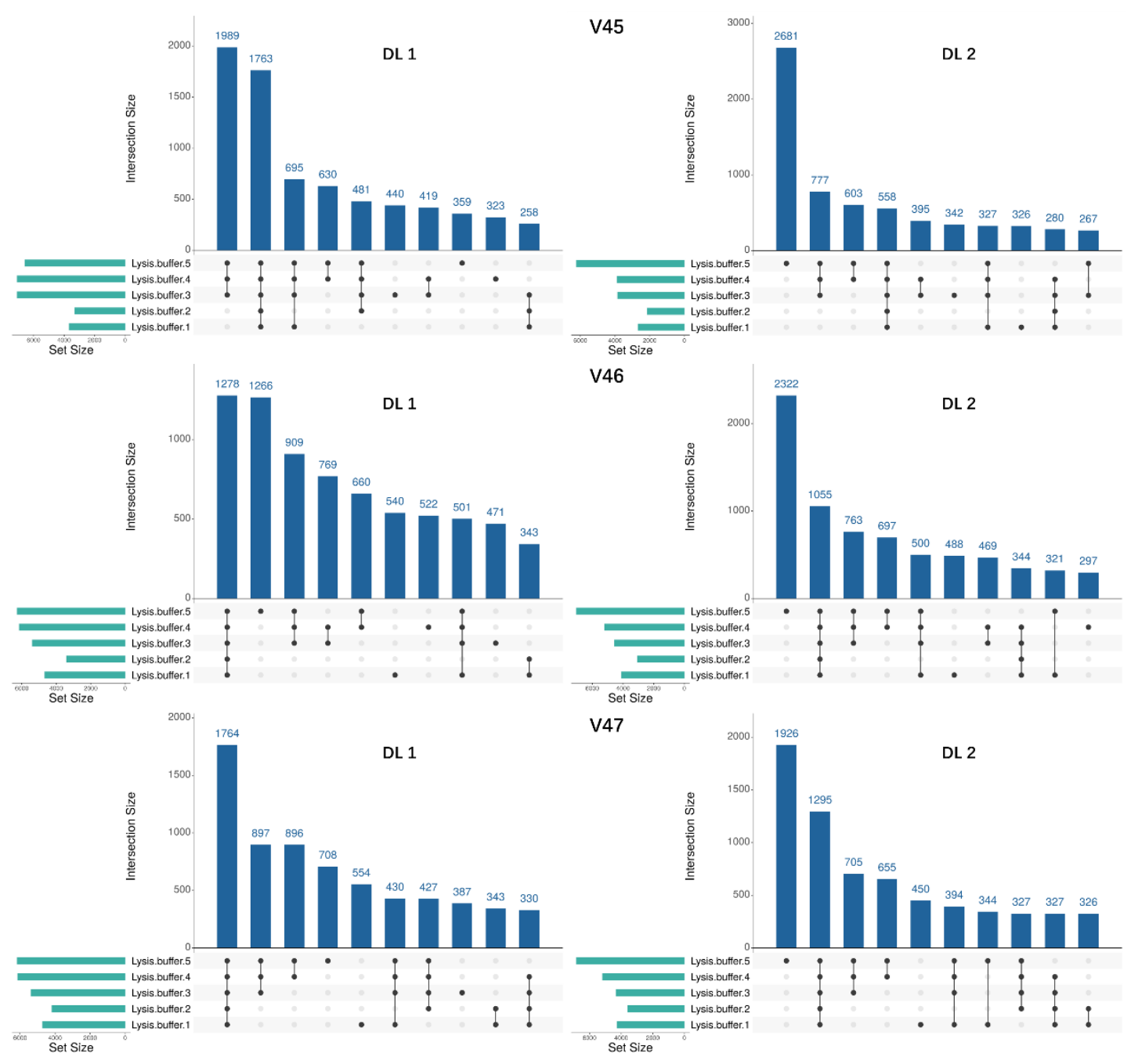

Figure S1. The overlap of protein groups identified among the five lysis conditions using DL1 and DL 2 


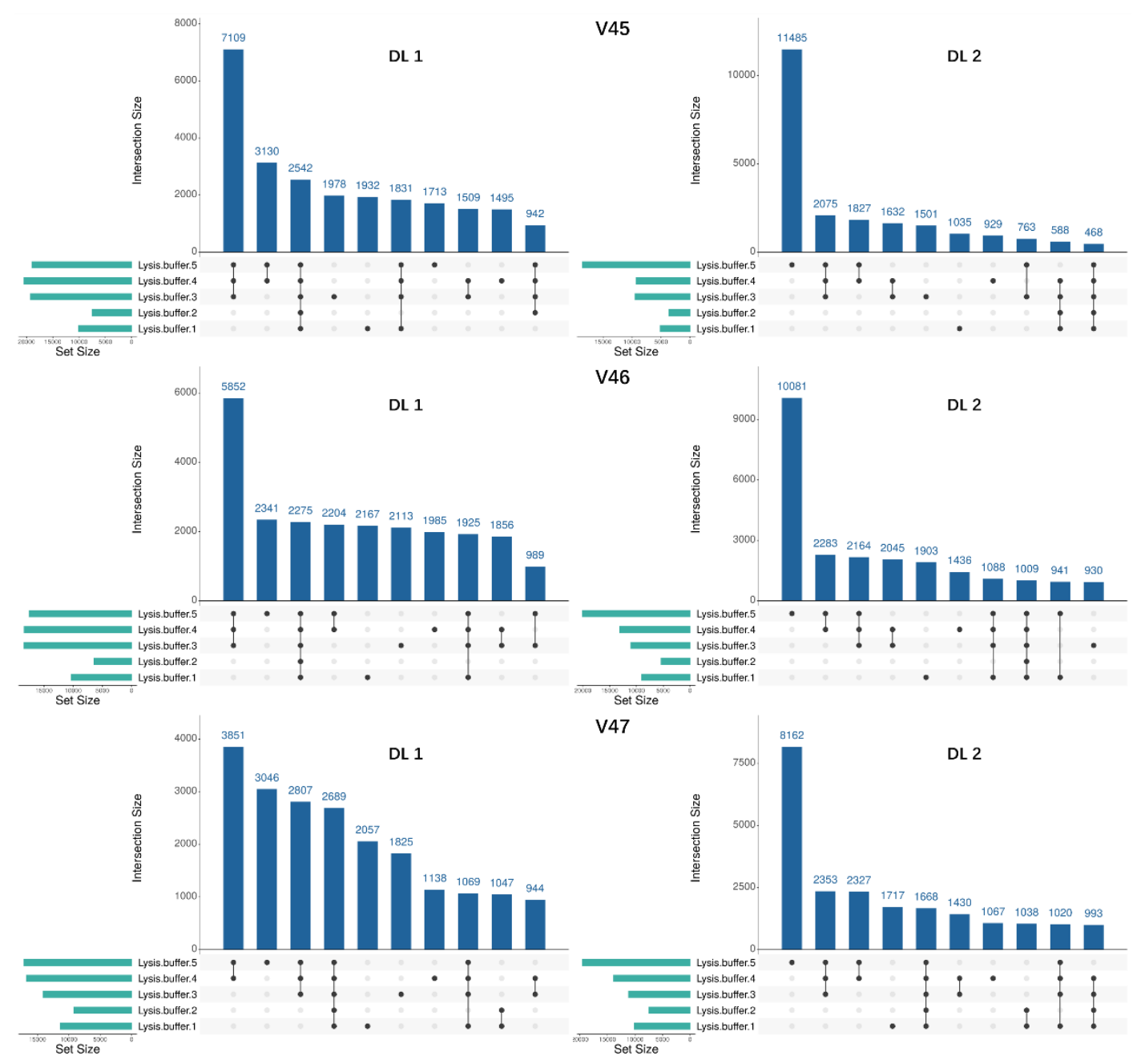

Figure S2. The overlap of peptides identified among the five lysis conditions using DL 1 and DL 2 

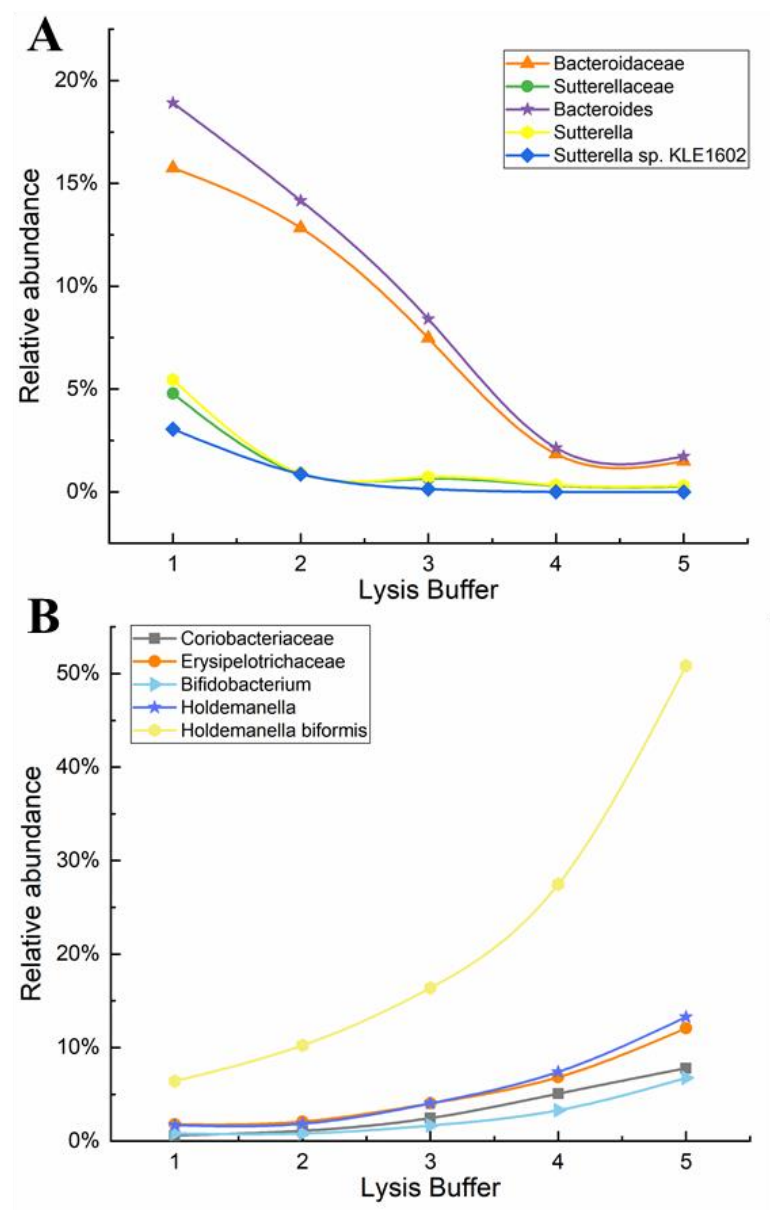

Figure S3. The change of relative abundance of several representative families, genera and species with DL 1. 

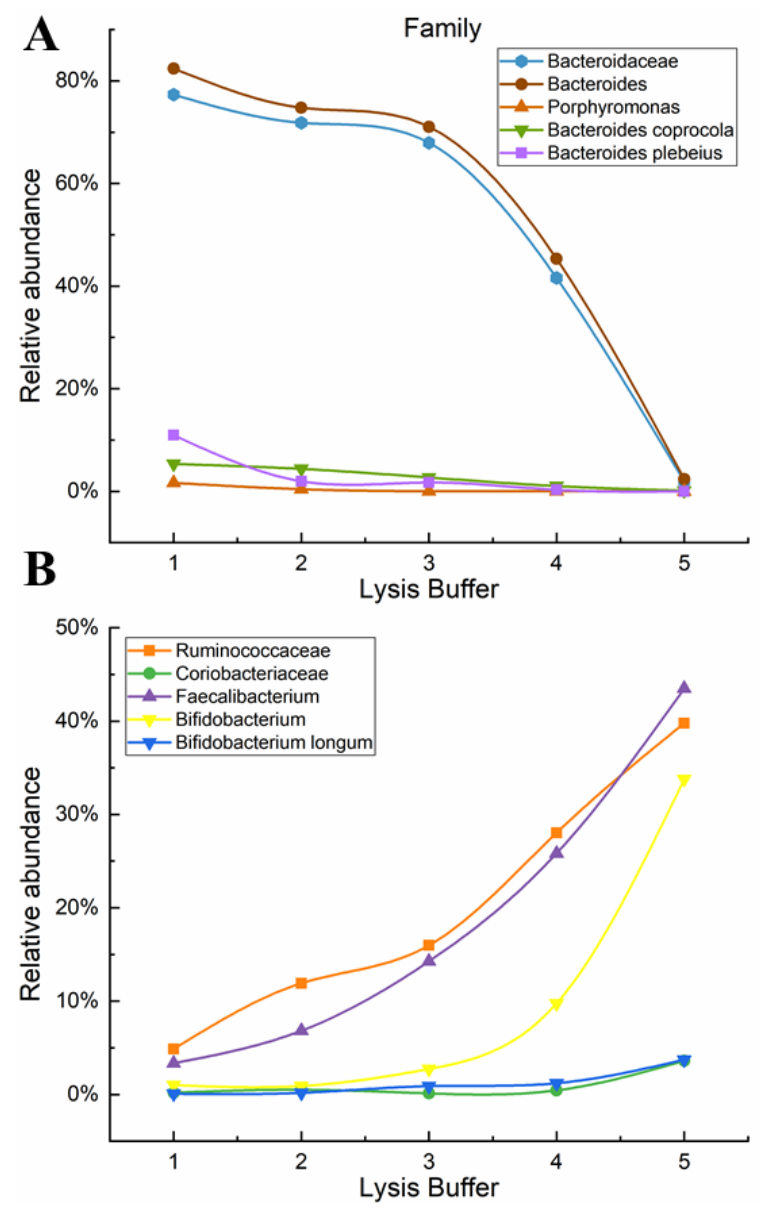

Figure S4. The change of relative abundance of several representative families, genera and species with DL 2. 


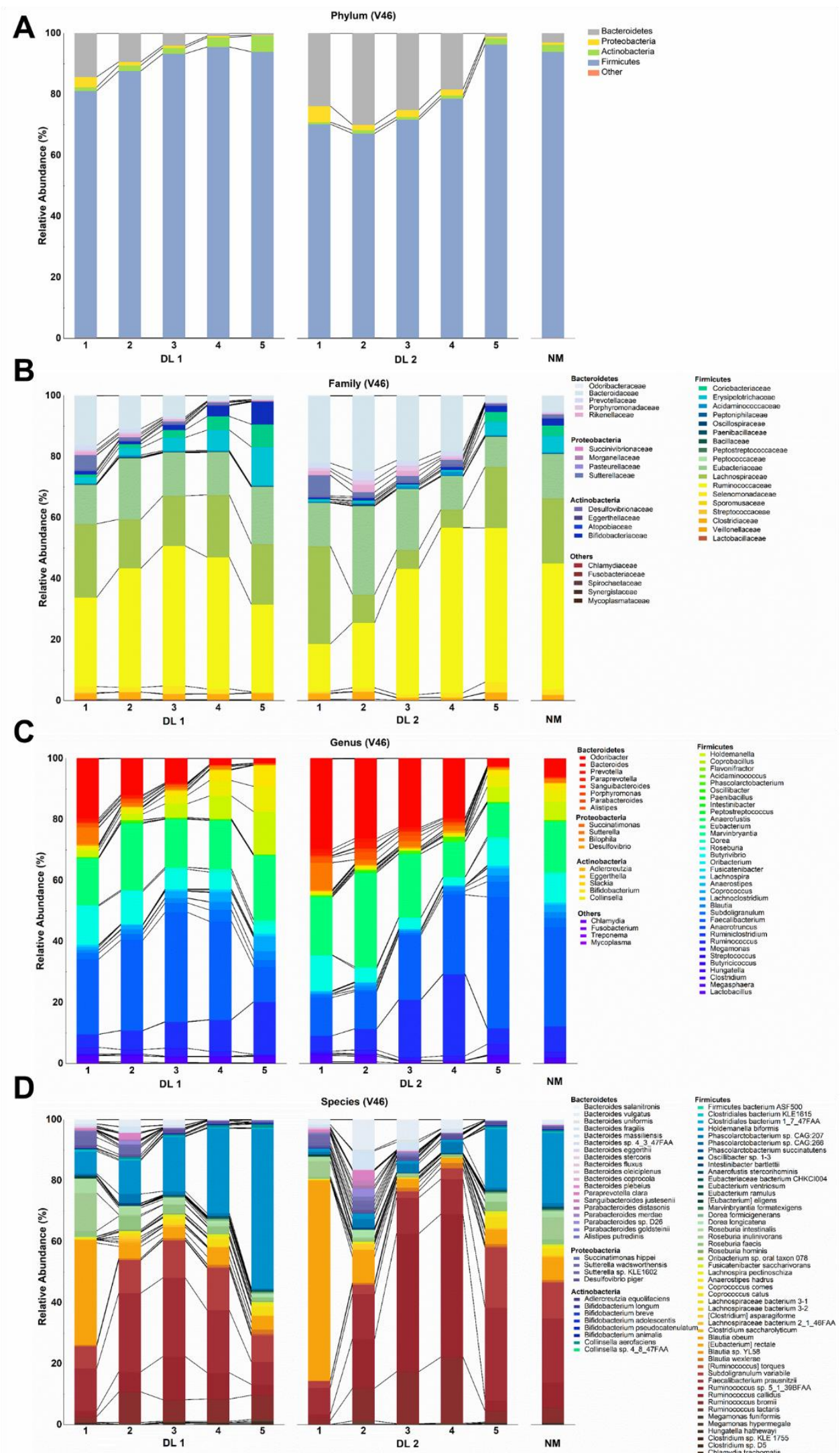

Figure S5. Evaluation of two differential lysis methods (DL 1 and 2) and normal method (NM) on the taxonomic compositions of the gut metaproteome in each step in sample V46. (A) Phylum level distributions; (B) Family level distributions; (C) Genus level distributions; (D) Species level distributions. 

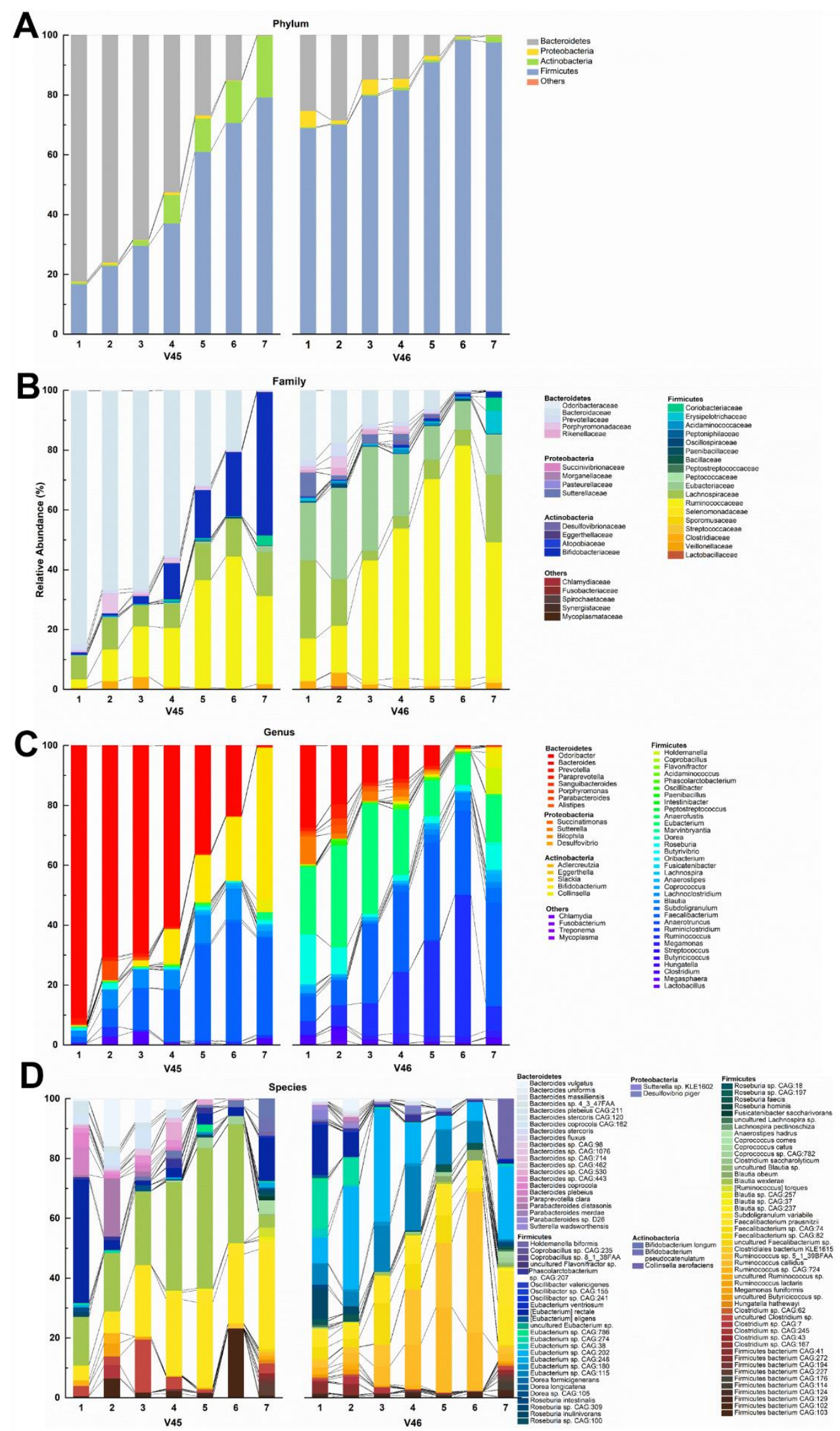

Figure S6. Evaluation of two differential lysis methods (DL 1and DL 2) and normal method (NM) on the taxonomic compositions of the gut metaproteome in each step in sample V47. (A) Phylum level distributions; (B) Family level distributions; (C) Genus level distributions; (D) Species level distributions. 

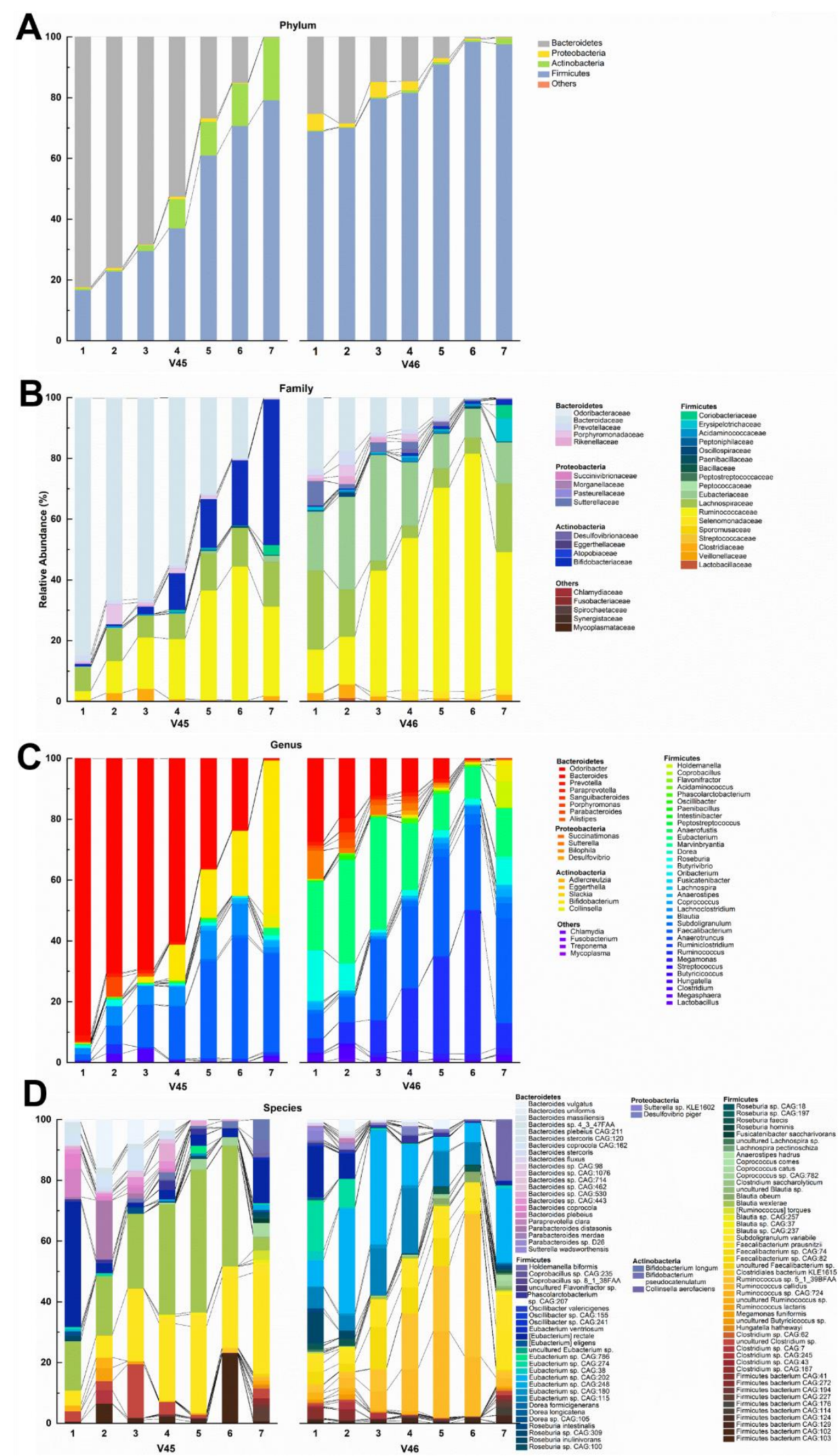

Figure S7. Evaluation of the seven-step protocol with DL 2 on the taxonomic compositions of the gut metaproteome in each step in sample V45 and V46. (A) Phylum level distributions; (B) Family level distributions; (C) Genus level distributions; (D) Species level distributions. 


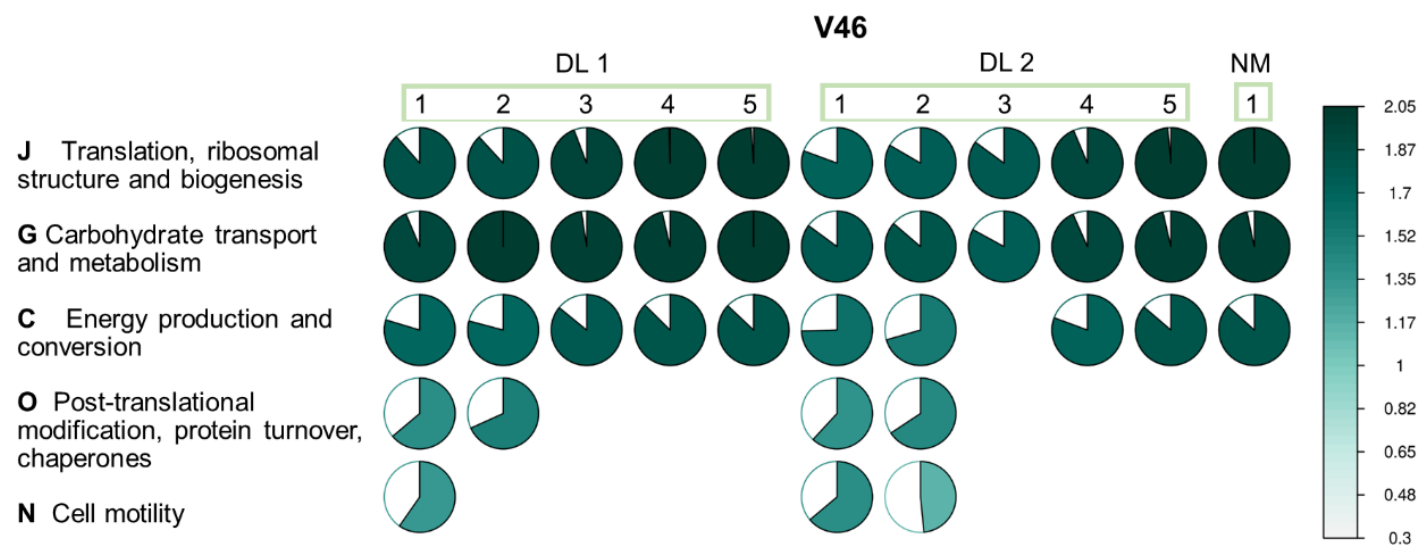

Figure S8. Evaluation of differential lysis methods DL 1 and DL 2 and the normal method (NM) on the distributions of COG categories based on the enrichment analysis in sample V46. Each row indicates a COG functional category, and each column indicates a lysate. The names of COG categories were indicated on the left side. The value of P-adjusted to filter the enrichment is $<0.01$. 


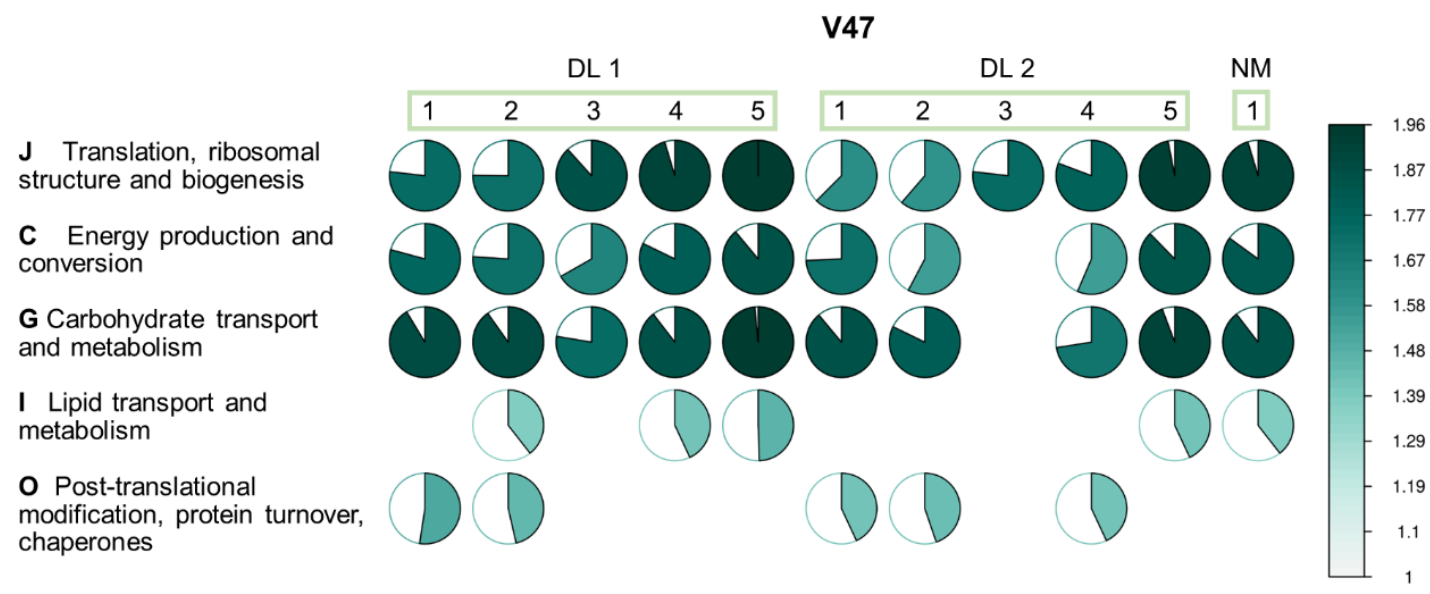

Figure S9. Evaluation of differential lysis methods DL 1, and 2 and normal method (NM) on the distributions of COG categories based on the enrichment analysis in sample V47. Each row indicates a COG functional category, and each column indicates a lysate. The names of COG categories were indicated on the left side. The value of Padjusted to filter the enrichment is $<0.01$. 